\title{
ATROFIAS CEREBRAIS EM ALCOÓLATRAS CRÔNICOS. ESTUDO PNEUMENCEFALOGRÁFICO
}

\author{
ORESTES BARINI* \\ Celso Pereira da Silva*
}

Nos alcoolistas crônicos encontramos, com certa freqüência, distúrbios mentais enquadráveis na síndrome psiquiátrica de tipo lesional deficitária do lobo frontal (apatia, deficiência de iniciativa prática, rebaixamento do senso crítico e da inteligência) e/ou crises convulsivas de ocorrência tardia. Relacionando êstes dados da observação clínica com os clàssicamente conhecidos da anatomia patológica, que evidenciam processos atróficos do parênquima nervoso nesses doentes, procuramos verificar a possibilidade de os documentar in vivo por meio da pneumencefalografia. Além do interêsse prático no diagnóstico clínico, esta comprovação teria valor médico-legal.

$\mathrm{Na}$ pesquisa bibliográfica que fizemos, abrangendo até o ano de 1953 inclusive, não encontramos trabalho algum a êsse respeito **.

\section{MATERIAL E MÉTODO}

O material provém do Serviço de Neuropsiquiatria do Hospital Militar da Fôrça Pública do Estado. Os pacientes foram internados para a terapêutica de delirium tremens, de crises convulsivas, de polineuropatias ou de distúrbios de conduta. Visamos exclusivamente a evidenciação de alteraçōes encefálicas por meio de pneumencefalografia.

Apresentamos os resultados da pneumencefalografia em 42 pacientes *** que fizeram uso imoderado de bebidas alcoólicas (aguardente de cana) por tempo supeperior a 10 anos. Todos os pacientes foram estudados do ponto de vista clínico, sendo excluídos os casos de moléstlas degenerativas pré-senis, de arteriosclerose cerebral, referência a traumatismos crânio-encefálicos e aquêles em que havia neurolues.

O exame pneumencefalográfico obedeceu à seguinte rotina: administraçāo de $0,2 \mathrm{~g}$ de Nembutal por via oral 30 minutos antes, e injeção intravenosa de Scofedal imediatamente antes do exame; punção suboccipital, com o paciente sentado, sendo

Trabalho do Servico de Neurologia da Escola Paulista de Medicina (Prof. Paulino W. Longo. * Assistente.

** Este trabalho foi terminado em 1955, sendo publicado agora aproveitando a oportunidade desta homenagem a Celso Pereira da Silva.

*** Parte do material (37 casos) constituiu motivo de comunicação ao Departamento de Neuro-Psiquiatria da Associação Paulista de Medicina (Rev. Paulista de Med., 45:417, outubro, 1954). 
retirado, fracionadamente, liqüido cefalorraquidiano em quantidade variável de 65 a $160 \mathrm{ml}$ (em média $120 \mathrm{ml}$ ), e injetada igual quantidade de ar; logo depois o paciente era pósto em decúbito, sendo feitas radiografias sagitais (occipito e frontoplaca) e de perfil (direito e esquerdo-placa); estando o paciente deitado.

\section{RESULTADOS}

Os resultados do craniograma simples foram normais, exceto em 4 pacientes que apresentavam sinais de discretas displasias. Os achados pneumencefalográficos — todos patológicos e exemplificados nas figuras 1 e 2 - foram resumidos no guadro 1.
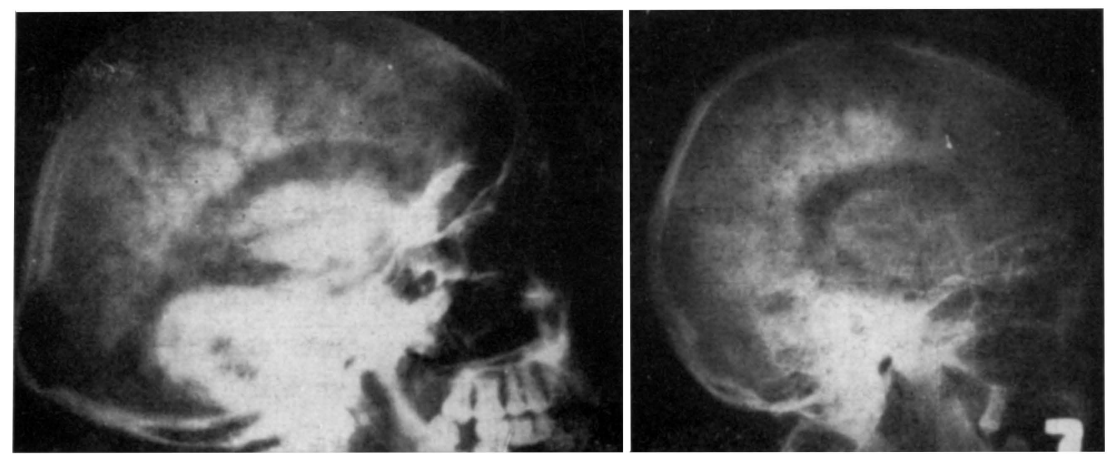

Fig. 1 - $A$ esquerda: dilatação ventricular de grau acentuado e simétrico; atrofia cortical difusa de grau médio (caso 37). Á direita: dilatação ventricular discreta; atrofia cortical difusa de grau médio (caso 25).
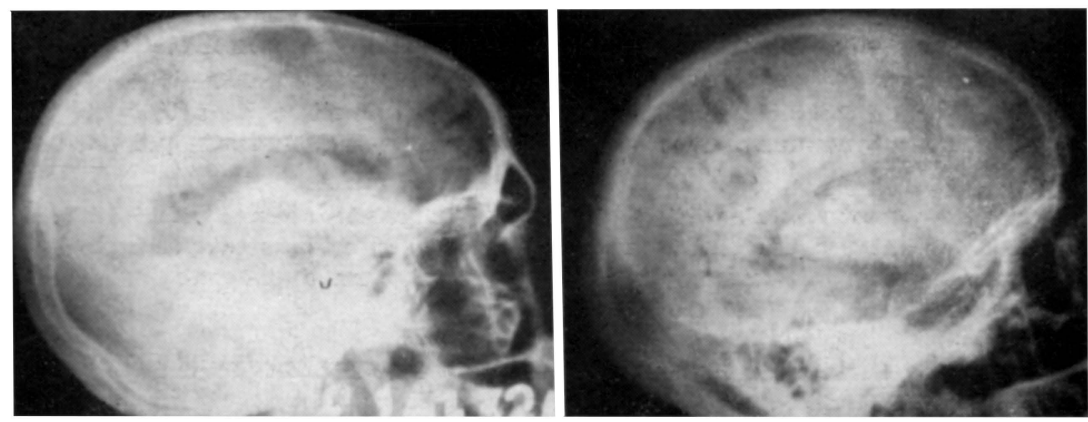

Fig. 2 - À esquerda: dilatação ventricular assimétrica mais acontuada no ventriculo lateral direito; escassa repleção cortical (caso 33). À direita: discreta dilatação ventricular; atrofia cortical de grau médio nas regióes frontal, parietal e temporal (caso 38). 


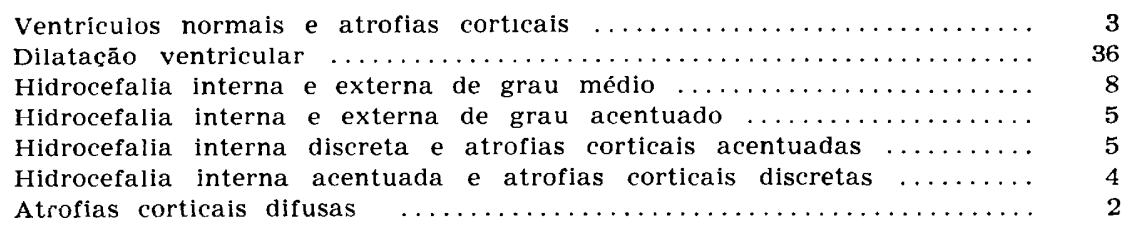

Atrofias corticais predominantes nas regiöes:

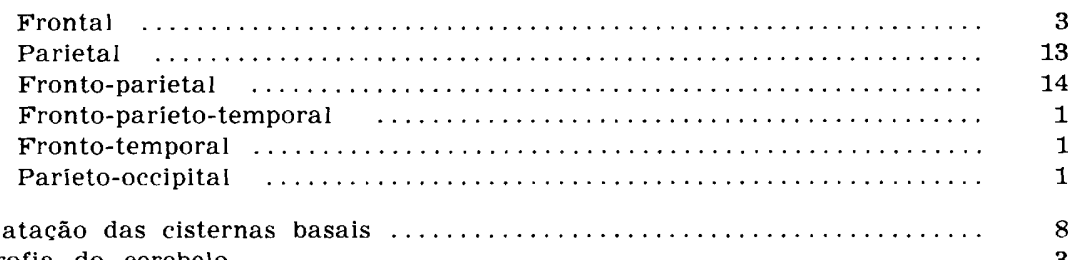

Atrofia do cerebelo $\ldots \ldots \ldots \ldots \ldots \ldots \ldots \ldots \ldots \ldots \ldots \ldots \ldots \ldots \ldots \ldots \ldots \ldots$

Quadro 1 - Resultados da pneumencefalografia em 42 alcoólatras crônicos.

\section{CONCLUSOES}

1. O estudo pneumencefalográfico de 42 pacientes que faziam uso imoderado e prolongado de bebida de alto teor alcoólico (aguardente de cana de açúcar) demonstrou, em todos, a existência de alterações atróficas do encéfalo.

2. Embora não seja possível afastar, em todos os 42 casos estudados, a ação eventual de outros fatôres determinantes de atrofia cerebral (particularmente fatôres disgenéticos), o conhecimento clássico anátomo-patológico da existência de atrofias cerebrais no alcoolismo crônico, associado à existência, em todos os cusos estudados, de quadro de atrofia cerebral, autoriza a atribuir à intoxicação alcoólica crônica as atrofias cerebrais encontradas.

3. Ao exame pneumencefalográfico deve ser atribuído relevante valor médico-legal na documentação de observações de alcoólatras crônicos.

\section{RESUMO}

Os autores consideram o exame pneumencefalográfico como capaz de documentar in vivo as lesões atróficas conseqüentes ao alcoolismo crônico descritas em Neuropatologia; apresentam os resultados de exames pneumencefalográficos feitos em 42 pacientes que faziam uso imoderado e prolongado de bebidas de alto teor alcoólico (aguardente de cana de açúcar), 
comprovando haver, em todos, aumento maior ou menor dos espaços ocupados pelo líqüido cefalorraquidiano.

Admitem os autores que, nos 42 casos estudados, a atrofia cerebral sobreveio em conseqüência da intoxicação alcoólica crônica, embora nāo seja possivel afastar, de todo, a possibilidade de ação de outros fatôres (particularmente disgenéticos) determinantes de atrofias cerebrais; assim, relevante valor médico-legal deve ser atribuido ao exame pneumencefalográfico na documentação de observações de alcoólatras crônicos.

\section{SUMMARY}

Brain atrophies in chronic alcoholism. Pneumoencephalographic study.

The authors present the pneumoencephalography as a method able to show in vivo the cerebral atrophic lesions resulting of chronic alcoholism. The results of pneumoencephalographic examinations in a series of 42 patients who have made immoderated and prolongated use of alcoholic beverages with high alcoholic contents (sugar-cane-liquor) are exposed: in all cases there was discreet or accentuated increase of the spaces occupied by cerebrospinal fluid. The authors suppose that in the 42 cases the cerebral atrophy was produced by chronic alcoholic intoxication although it is impossible to eliminate the possibility that other factors (especially disgeneticals) could be responsible by such atrophy; they attribute a high medical-legal value to pneumoencephalography for the documentation of observations of chronical alcoholics.

Serviço de Neurologia da Escola Paulista de Medicina - Caixa Postal $5496-$ São Paulo, Brasil. 